\title{
Iterated Belief Revision In the face of Uncertain Communication
}

\author{
Yoshitaka Suzuki ${ }^{1}$, Satoshi Tojo ${ }^{1}$, and Stijn De Saeger ${ }^{2}$ \\ 1 Japan Advanced Institute of Science and Technology \\ \{syoshita,tojo\} @jaist.ac.jp \\ 2 National Institute of Information and Communications Technology \\ stijnenict.go.jp
}

\begin{abstract}
This paper offers a formalization of iterated belief revision for multiagent communication using the logic of communication graphs introduced in [15]. In this study we consider an agent (i.e., information source) capable of sending two types of message. In the first type, he tells that he knows a proposition, but in the second type, he tells that he believes a proposition. Consequently, iterated belief revision is brought about through a sequence of communication events (i.e., a history), and we propose a variation of the AGM rational postulates for history based belief revision. As we will show, a representation theorem is verified only for a class of restricted communication graphs. We consider this result to be a weak point in the application of the AGM postulates to multiagent communication, and propose a viable alternative.
\end{abstract}

\section{Introduction}

Information processing in uncertain communication environments is one of the important problems for the study of multiagent systems. When agents communicate, often less than certain information passes between them. In such a case, we technically cannot say that they acquire knowledge, even if the information in question eventually turns out to be true. Here, we distinguish between two types of uncertain information in multiagent communication. On the one hand, an agent may consider a given piece of information correct even though in fact he obtains it from an unreliable information source (via some insecure communication channel), and therefore his information may in fact be incorrect. On the other hand, the agent may not be convinced that this information is correct, but nonetheless consider it plausible. While he does not recognize it in the first case, he is aware of his own uncertainty in the second case. Thus, making an inquiry to some other agent, one agent finds two fallible answers. That is to say, the other agent is firmly convinced of the information or not ${ }^{3}$. These two types of epistemic attitudes, related to knowledge and belief, require a classification of rational processing for uncertain information. Traditional study of belief revision $([1,7])$ proposes rational postulates for the change of an agent's knowledge base called minimal change, and

\footnotetext{
${ }^{3}$ For the purpose of this paper, we take 'conviction' to be a technical notion that is epistemically stronger than belief, excluding the possibility of error in the agent's own mind. Thus, whereas introspectively an agent does not distinguish between his convictions and knowledge, he would consider his beliefs to be likely though not certain.
} 
prove a representation theorem that tells us that these postulates correspond to certain revision operators. However, Gärdenfors ([7]) stated that the relation between knowledge base and external environment is not essential for the study of belief revision, and he did not distinguish knowledge from belief. Therefore, when a message comes from the other agent, whether the other agent knows something or not is not important for the general study of belief revision.

As an exception to the above characterization, Friedman and Halpern ([5, 6]) defined a belief operator in terms of both a knowledge operator and a conditional operator. Their study proposed an expansion of the logic of knowledge for multiagent systems [4], and therefore it can be applied to multiagent systems, although they were interested in iterated belief revision for single agents. However, they assumed that an agent acquires an infallible information by the observation upon the external environment. As a result, any given sequence of external inputs must be consistent, and possible worlds that are inconsistent with the inputs already accepted are systematically eliminated. However, as indicated by Rott's study about conservatism in iterated belief revision [16], such an idea is radical for fallible information. Moreover, since they were not interested in communication, their model must not be related with the study of multiagent system. Because multiple agents' communication may give rise to uncertain information, such a formalization is suitable for single agents, but not multiple agents. In fact, they did not describe the application of the framework to multiagent systems. Thus, the traditional study of belief revision usually assumed that epistemic input came from an external environment, and was generally not interested in multiagent communication.

Studying belief revision in multiagent communication, we utilize the logic of communication graphs from [15]. This logic expresses that information travels via communication channels that ensure its reliability. Only if an agent $i$ can directly receive some piece of information from another agent $j$ and $j$ has acquired this knowledge in a similar fashion, $i$ acquires this piece of knowledge from $j$. Nevertheless, this logic cannot represent the situation where an agent believes some proposition but does not know it. In order to solve the problem, we will not directly use the modal operator $K_{i}$ for knowledge. Instead, we introduce the operator $C_{i}$ for conviction and $\rightarrow_{i}$ for conditional, and define knowledge $K_{i}$ and belief $B_{i}$ in terms of these two operators. Furthermore, we will define some postulates for belief revision operations that accepts another agent's respective knowledge and beliefs as external inputs, and show that some revision operators are equivalent with these postulates when the communication graph satisfies some condition.

In section 2, we introduce formal preliminaries and semantics for our logic of communication graphs that pays attention to the other agent's knowledge and belief. In section 3, consulting moderate and radical belief revision in Rott [16], we define iterated revision operators for knowledge and belief. In section 4, rational postulates are proposed, and we show the representation theorem between the operators and the postulates for those cases where the communication graph satisfies some particular constraints. In section 5, we discuss some remaining problems of our study and its perceived advantages over related works, as well as indicate subsequent work to be done. 


\section{Preliminaries}

In this section, we describe the semantics for our logic of communication graphs with conditionals $\mathcal{C C}(\mathcal{G})$. This is based on the logic of communication graphs from [15], expanded with a conditional operator like the study of belief revision by the logic of knowledge for multiagents by $[5,6]$. Thus our logic not only allows reasoning about the underlying communication channel, but also represents the plausibility of a given proposition as far as the agents are concerned.

Let $\mathcal{A}$ be the set of agents. Then, a communication graph is described by $\mathcal{G}_{\mathcal{A}}=$ $\langle\mathcal{A}, E\rangle$, where $E \subseteq(\mathcal{A} \times \mathcal{A}) \backslash\{(i, i) \mid i \in \mathcal{A}\}$. The edges in $E$ represent whether an agent can directly receive information from another agent or not, i.e., $(i, j) \in E$ means that agent $i$ learns information from agent $j$ directly.

We define that $E$ is fully connected iff for all $i, j \in \mathcal{A}$, if $i \neq j$, then $(i, j) \in E$, and $E$ is existentially connected iff for all $i \in \mathcal{A}$, for some $j \in \mathcal{A},(i, j) \in E$. In the former case, any agent can communicate with any agent. In the latter case, for any agent, there is an information source.

Suppose that all agents share the (finite) set of atomic propositions $A t$ and a special propositional variable $L$. Intuitively, $L$ means that any information exchange have emerged via reliable communication channel. As already discussed, we introduce the following modal operators; intuitively, $C_{i} \phi$ means that $i$ is convinced that $\phi ; \phi \rightarrow_{i} \psi$ means that, given $\phi, \psi$ is plausible for $i$; $\diamond \phi$ means that, after some communications, $\phi$ becomes true. Precise semantics of these symbols are given later. The set of well-formed formulae consists of

$$
\phi:=p|\neg \phi| \phi \wedge \psi\left|C_{i} \phi\right| \phi \rightarrow_{i} \psi \mid \diamond \phi
$$

We use the standard abbreviations for connectives $\vee, \Rightarrow, \Leftrightarrow$, and propositional constants true and false. Besides these we introduce the modal operators $K_{i}$ and $B_{i}$ as abbreviations, where as usual $K_{i} \phi$ means that $i$ knows $\phi$, and $B_{i} \phi$ means that $i$ believes $\phi$.

$$
\begin{gathered}
K_{i} \phi \stackrel{\text { def }}{=} L \wedge C_{i} \phi \\
B_{i} \phi \stackrel{\text { def }}{=} C_{i}\left(\text { true } \rightarrow_{i} \phi\right)
\end{gathered}
$$

Thus, agent $i$ knows $\phi$ iff any information exchange have emerged via reliable communication channel and agent $i$ is convinced that $\phi$. Agent $i$ believes $\phi$ iff agent $i$ is convinced that $\phi$ is plausible.

The definition of $B_{i}$ is almost the same as Friedman and Halpern's in [5, 6], but in their work belief is defined instead as

$$
B_{i} \phi \stackrel{\text { def }}{=} K_{i}\left(\text { true } \rightarrow_{i} \phi\right) .
$$

That is to say, agent $i$ believes $\phi$ iff agent $i$ knows that $\phi$ is plausible. Their definition of knowledge is not based on communication channel, but our definition depends on the reliability of the communication channel, which is decided by an agent's external 
environment (See the definition of the semantics of $L$ below) ${ }^{4}$. Thus, whether he knows something or not is not decided by his internal state without external environment. However, belief does not seem to depend on the external environment. Therefore, we introduced modal operator $C_{i}$ that does not depend on the real external environment, but on the environments that cannot be distinguished from the real environment for the agent $i$. For details, see the definition fo the semantics of $C_{i}$.

Let $A t_{i} \subseteq A t$ be the set of atomic propositions of which agent $i$ initially knows the truth value (we do not assume all $A t_{i}(i \in \mathcal{A})$ to be disjoint). Furthermore, this fact about $i$ 's knowledge is common knowledge among all members of $\mathcal{A}$. So another agent knows that $i$ knows the truth value of elements of $A t_{i}$, though not what these values actually are. Thus we introduce an information vector $\boldsymbol{A} \boldsymbol{t}=\left\langle A t_{1}, \ldots, A t_{n}\right\rangle$ representing the initial knowledge of the agents. Given a set $W$, we say that $v \in W$ is a world, where $v$ is a function $v: A t \rightarrow\{0,1\}$.

When agent $i$ learns some information $\phi$ from agent $j$ 's knowledge or beliefs, we represent this situation by $(i, j, \phi, e)$ and call it a communication event, where $e \in\{B, K\}$ is a label representing the epistemic modality in question. Technically $\phi$ is restricted to formulae in disjunctive normal form (DNF), i.e., $\phi$ is of the form $\bigvee_{i=1, \ldots, k} \wedge C_{i}$, where each $C_{i}$ is a consistent finite set of literals in $A t$. That is, $(i, j, \phi, K)$ is an event that agent $i$ learns from agent $j$ that $j$ knows $\phi$. Given the set of all communication events $\Sigma_{\mathcal{G}}, H \in \Sigma_{\mathcal{G}}^{*}$ is a finite sequence of events called a history, where the empty history is denoted $\epsilon$. We define the temporal ordering over all histories as follows: $H \sqsubseteq H^{\prime}$ iff $H^{\prime}=H \cdot H^{\prime \prime}$ for some $H^{\prime \prime}$, where · is a concatenation of communication events.

A history can be considered as a God's eye record of past agent communications, but an agent may only witness part of it. When the second agent $j$ and the third agent $k$ communicate with each other, the first agent $i$ has no access to their communication. Therefore, we introduce $i$ 's local history $\lambda_{i}(H)$, in which $i$ can only recognize those events that concern himself, i.e., in which $i$ receives some information from the other agent. It is defined as follows.

$$
\lambda_{i}(H \cdot(m, j, \phi, e))= \begin{cases}\lambda_{i}(H) \cdot(m, j, \phi, e) & \text { if } m=i \\ \lambda_{i}(H) & \text { otherwise }\end{cases}
$$

A pair $(v, H)$ consisting of a system state $v$ and a history $H$ is called a point. Given two points $(w, H)$ and $\left(v, H^{\prime}\right)$, we can imagine an agent $i$ unable to distinguish between the two. We can describe this situation in terms of an accessibility relation $\sim_{i}$ as follows, where $\left.w\right|_{A t_{i}}$ is a restriction of $w$ whose domain is $A t_{i}$.

$$
(w, H) \sim_{i}\left(v, H^{\prime}\right) \text { iff }\left.w\right|_{A t_{i}}=\left.v\right|_{A t_{i}} \text { and } \lambda_{i}(H)=\lambda_{i}\left(H^{\prime}\right)
$$

\footnotetext{
${ }^{4}$ In the following discussion, predicate $L$ is defined by communication channel. Whether an agent knows something or not is justified by $L$. In other words, the source of the knowledge lies outside of himself. Thus, in our formalization, an agent has no access to the ground of the justification directly. Philosophically, such a view is called externalism about knowledge [2].
} 
We use a total preorder $\leq{ }^{5}$ over $W$ for the definition of conditional operator and we call it a preference relation. We denote $v \leq w$ when $v$ is at least as plausible as $w$. $v<w$ is the strict case of $\leq$, i.e., $v \leq w$ and $w \not v$. We assume that sets of states are also comparable with $\leq$. Thus, $A \leq B$ iff for all $v \in B$, for some $w \in A, w \leq v$. The maximal worlds w.r.t. the preference relation are called implausible worlds. The set of implausible worlds $I m \subseteq W$ satisfies the following condition: for any $w \in I m$, $v \in W, v \leq w$, and if $v \notin I m$, then $w \not z$. Let a preference assignment function $\mathcal{P}_{i}$ for agent $i$ be a mapping from $(v, H)$ to $(\leq, I m)$, i.e., $\mathcal{P}(v, H)=(\leq, I m)$, where $\operatorname{Im}=\left\{w \in W \mid\right.$ for any $H^{\prime},\left(w, H^{\prime}\right) \chi_{i}(v, H)$ or $\left.w, H^{\prime} \forall \forall_{\mathcal{M}} L\right\}\left(\models_{\mathcal{M}}\right.$ is precisely defined in the following discussion). That is to say, implausible worlds can be distinguished from the actual world in which agent $i$ finds himself. In the following discussion, we define $\mathcal{P}_{i}(v, H)=\left(\leq_{i, v, H}, \operatorname{Im}_{i, v, H}\right)$. That is, $w \leq_{i, v, H} w^{\prime}$ means that $w$ is at least as plausible as $w^{\prime}$ for agent $i$ at world $v$ and history $H$. Thus, we will define a communication graph model as a tuple $\mathcal{M}=\langle\mathcal{G}, \boldsymbol{A t}, \mathcal{P}, W\rangle$.

We will introduce the satisfiability relation of legality and truth as follows.

(Legality)

- $w, \epsilon=\mathcal{M} L$

- $w, H \cdot(i, j, \phi, K) \models_{\mathcal{M}} L$ iff $w, H \models_{\mathcal{M}} L,(i, j) \in E$, and $w, H \models_{\mathcal{M}} K_{j} \phi$

- $w, H \cdot(i, j, \phi, B) \models_{\mathcal{M}} L$ iff $w, H \models_{\mathcal{M}} L$ and $(i, j) \in E$

(Truth)

- $w, H \mid=_{\mathcal{M}} p$ iff $w(p)=1$, where $p \in A t$.

- $w, H={ }_{\mathcal{M}} \neg \phi$ iff $w, H \mid \neq_{\mathcal{M}} \phi$

- $w, H \mid=_{\mathcal{M}} \phi \wedge \psi$ iff $w, H \models_{\mathcal{M}} \phi$ and $w, H \models_{\mathcal{M}} \psi$

- $w, H==_{\mathcal{M}} \diamond \phi$ iff $\exists H^{\prime}$ such that $H \sqsubseteq H^{\prime}, w, H^{\prime} \models_{\mathcal{M}} L$ and $w, H^{\prime} \models_{\mathcal{M}} \phi$

- $w, H={ }_{\mathcal{M}} C_{i} \phi$ iff $\exists\left(v, H^{\prime}\right),(w, H) \sim_{i}\left(v, H^{\prime}\right)$ and $v, H^{\prime} \models_{\mathcal{M}} L$, and $\forall\left(v, H^{\prime}\right)$, if $(w, H) \sim_{i}\left(v, H^{\prime}\right)$ and $v, H^{\prime} \mid=_{\mathcal{M}} L$, then $v, H^{\prime} \models_{\mathcal{M}} \phi$

- $w, H=_{\mathcal{M}} \phi \rightarrow_{i} \psi$ iff $\models_{\mathcal{M}} \phi \Leftrightarrow$ false or $\llbracket \phi \wedge \psi \rrbracket_{i, w, H}^{\mathcal{M}}<_{i, w, H} \llbracket \phi \wedge \neg \psi \rrbracket_{i, w, H}^{\mathcal{M}}$, where $\llbracket \phi \rrbracket_{i, w, H}^{\mathcal{M}}=\left\{v \in W \mid \exists H^{\prime},(w, H) \sim_{i}\left(v, H^{\prime}\right)\right.$, and $v, H^{\prime} \models_{\mathcal{M}}$

$L$, and $\left.v, H^{\prime} \models \mathcal{M} \phi\right\}$.

We will explain the meaning of the above definition. Legality is defined as follows. At first, when a history is empty, the point is legal. Next, If there is some legal point, there is some communication graph from agent $j$ to agent $i$, and $j$ knows $\phi$, the pair of the world and the concatenation of the history and the event $(i, j, \phi, K)$ is also legal. Finally, if there is some legal point, and there is some communication channel from agent $j$ to agent $i$, then the pair of the world and the new history with $(i, j, \phi, B)$ is also legal. Thus, legality means that there have been reliable communication channels.

Note that agent $j$ 's knowledge is related with the definition of legality, but not $j$ 's belief, since legality is a condition that ensures the truth of knowledge (see the definition of $K_{i} \phi$ ). While knowledge must be justified by a reliable information source, whether

\footnotetext{
${ }^{5}$ A total preorder $\leq$ is a total and transitive relation. $\leq$ is total iff $w \leq w^{\prime}$ or $w^{\prime} \leq w$ for any $w, w^{\prime} \in W . \leq$ is transitive iff for any $w, w^{\prime} \in W$, if $w \leq w^{\prime}$ and $w^{\prime} \leq w^{\prime \prime}$, then $w \leq w^{\prime \prime}$.
} 
belief needs such a source or not is a difficult epistemological problem for our current study. Therefore, our current stance is that we do not require belief to be justified by legality.

The truth condition of $\neg$ and $\wedge$ is as usual. The formula $\diamond \phi$ is satisfied when there will be some future point that satisfies $\phi$. The formula $C_{i} \phi$ is true when there is some legal point that is indistinguishable with the real point for agent $i$, and for any indistinguishable legal point, $\phi$ is true. The formula $\phi \rightarrow_{i} \psi$ means that $\phi \wedge \psi$ is more plausible for agent $i$ than $\phi \wedge \neg \psi$ at the point.

The difference in definition between our $C_{i}$ and Pacuit and Parikh's $K_{i}$ in [15] is important. Their definition is as follows.

$w, H \models_{\mathcal{M}} K_{i} \phi$ iff $\forall\left(v, H^{\prime}\right)$, if $(w, H) \sim_{i}\left(v, H^{\prime}\right)$ and $v, H^{\prime} \models_{\mathcal{M}} L$, then $v, H^{\prime} \models_{\mathcal{M}} \phi$

That is, their definition of knowledge does not assume that there is some legal point that is not distinguished from the real points for agent $i$. This definition does not satisfy the modal axiom D, which characterizes an essential property of belief the property of belief (i.e., nobody believes a contradiction.), while the axiom $\mathrm{T}$ is known from the logic of knowledge (i.e., veridicality of knowledge). Perhaps the real point may not be legal, and we may not find any legal point that is not distinguished from the real points for agent $i$. In such a case, $K_{i}$ does not satisfy $D$. Therefore, we define $C_{i}$ as already mentioned.

Thus, the derived modal operators $K_{i}$ and $B_{i}$, defined by $L, C_{i}$, and $\rightarrow_{i}$, satisfy the following traditional axioms.

$\mathrm{K}:\left(K_{i} \phi \wedge K_{i}(\phi \Rightarrow \psi)\right) \Rightarrow K_{i} \psi$

$\mathrm{T}: K_{i} \phi \Rightarrow \phi$.

4: $K_{i} \phi \Rightarrow K_{i} K_{i} \phi$.

5: $\neg K_{i} \phi \Rightarrow K_{i} \neg K_{i} \phi$.

Gen: From $\phi$ infer $K_{i} \phi$.

$\mathrm{K}:\left(B_{i} \phi \wedge B_{i}(\phi \Rightarrow \psi)\right) \Rightarrow B_{i} \psi$.

D: $\neg B_{i}$ false.

4: $B_{i} \phi \Rightarrow B_{i} B_{i} \phi$.

5: $\neg B_{i} \phi \Rightarrow B_{i} \neg B_{i} \phi$.

Gen: From $\phi$ infer $B_{i} \phi$.

Note that $C_{i}$ also satisfies KD45 like $B_{i}$.

$\mathrm{K}:\left(C_{i} \phi \wedge C_{i}(\phi \Rightarrow \psi)\right) \Rightarrow C_{i} \psi$.

D: $\neg C_{i}$ false.

4: $C_{i} \phi \Rightarrow C_{i} C_{i} \phi$

5: $\neg C_{i} \phi \Rightarrow C_{i} \neg C_{i} \phi$.

Gen: From $\phi$ infer $C_{i} \phi$.

Furthermore, the conditional operator $\rightarrow_{i}$ satisfies the following axioms of nonmonotonic reasoning in $[12,13]$. 

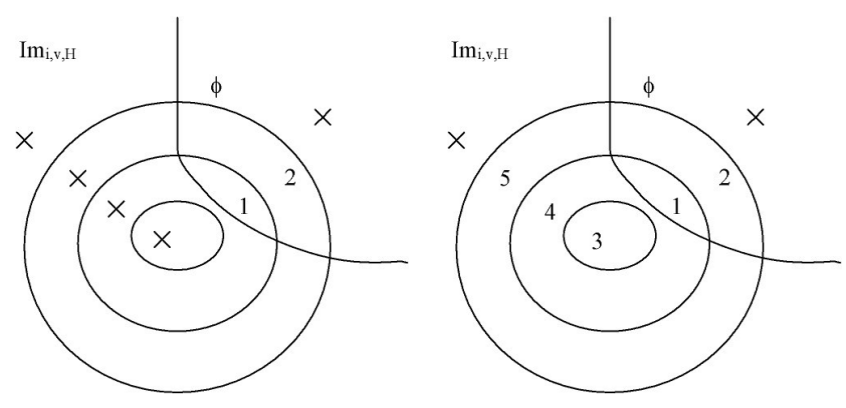

Fig. 1. Rewriting sphere by knowledge and belief

LLE: From $\phi \Leftrightarrow \phi^{\prime}$ and $\phi \rightarrow_{i} \psi$ infer $\phi^{\prime} \rightarrow_{i} \psi$.

RW: From $\psi \Rightarrow \psi^{\prime}$ and $\phi \rightarrow_{i} \psi$ infer $\phi \rightarrow_{i} \psi^{\prime}$.

REF: $\phi \rightarrow_{i} \phi$.

AND: From $\phi \rightarrow_{i} \psi_{1}$ and $\phi \rightarrow_{i} \psi_{2}$ infer $\phi \rightarrow_{i} \psi_{1} \wedge \psi_{2}$.

OR: From $\phi_{1} \rightarrow_{i} \psi$ and $\phi_{2} \rightarrow_{i} \psi$ infer $\phi_{1} \vee \phi_{2} \rightarrow_{i} \psi$.

CM: From $\phi \rightarrow_{i} \psi_{1}$ and $\phi \rightarrow_{i} \psi_{2}$ infer $\phi \wedge \psi_{1} \rightarrow_{i} \psi_{2}$.

RM: From $\phi \rightarrow_{i} \psi_{1}$ and not $\phi \rightarrow_{i} \neg \psi_{2}$ infer $\phi \wedge \psi_{2} \rightarrow_{i} \psi_{1}$.

Finally, our formalization satisfies the following interaction between knowledge and beliefs characterized by [11].

$\mathrm{KB} 1: B_{i} \phi \Rightarrow K_{i} B_{i} \phi$.

$\mathrm{KB} 2: K_{i} \phi \Rightarrow B_{i} \phi$.

In the following discussion, we will suppose that if $\left.w\right|_{A t_{i}}=\left.v\right|_{A t_{i}}$, then $\leq_{i, w, \epsilon}=\leq_{i, v, \epsilon}$, although the above axioms are satisfied without the supposition.

\section{Rewriting Rules for the Preference Relation}

In this section we introduce our iterated belief revision operation by incorporating the other agent's knowledge (or correctly the information that the agent regards as knowledge) and belief. Although this is basically identical to traditional sphere semantics by $[8,10]$, much of our idea depends on the radical/moderate approach to iterated belief revision by $[16]^{6}$, and therefore, we can not only revise one agent's belief by one input, but also by a sequence of inputs from the other agent's knowledge and belief. Namely, we revise an agent's belief not only by an input but rather by a sequence of inputs. The change of belief corresponds to that of preference relation. Thus, we will define rewriting rules for the preference relation.

\footnotetext{
${ }^{6}$ Rott studied the conservative approach to iterated belief revision in [16]. In this approach, when $\neg \psi \in K \dot{+} \phi,(K \dot{+} \phi) \dot{+} \psi=K \dot{+} \psi$, where $\dot{+}$ is a revision function for a set $K$ of formulae and a formula $\phi$. The radical/moderate approach does not satisfy such a property. For details, see [16].
} 
When we incorporate the information that states that $j$ knows $\phi$ into $i$ 's belief, we consider that the worlds which satisfy $\neg \phi$ become implausible for agent $i$, because knowledge must be true. Perhaps, this information may be false due to $j$ 's misjudgment, when his information was acquired from an unreliable information source. However, we will consider that $i$ relies on $j$ 's information if $i$ can assume that he finds himself at some legal point and $j$ has acquired the information in question from a reliable source. Thus, we use the radical approach for the definition of revision by another agent's knowledge. This approach is also presupposed by the study of belief revision in modal logic [5, 6], where worlds that contradict the input are systematically deleted. Such a method is suitable for information that is known (i.e. guaranteed to be correct), because any situation that contradicts it should be eliminated from the set of worlds still considered possible.

The basic idea can be intuitively described by systems of spheres like the left side of figure 1. Spheres represent the various equivalence classes imposed on $W$ by the preference relation $\leq_{i, v, H}$. Therefore, implausible worlds are at the outside of all the spheres. Thus, change of preference relation by knowledge is explained as follows: the most plausible worlds that satisfy $\phi$ (worlds of part 1 in the figure) become the most plausible worlds after accepting the knowledge that $\phi$, the second plausible worlds that satisfy $\phi$ (worlds of part 2 in the figure) become the second-most plausible worlds in the next step, but implausible worlds that satisfy $\phi$ and any worlds that do not satisfy $\phi$ (worlds with black marks) become implausible worlds in the next step.

More formally, $w \leq_{i, v, H \cdot(m, j, \phi, K)} w^{\prime}$ iff

I. $v, H \neq_{\mathcal{M}} L$ or $(m, j) \notin E$ or $v, H \models_{\mathcal{M}} \neg K_{j} \phi$ or

II. $m \neq i$ and $w \leq_{i, v, H} w^{\prime}$ or

III. $m=i$ and

a. $w^{\prime} \in I m_{i, v, H \cdot(i, j, \phi, K)}$ or

b. $w \notin I m_{i, v, H \cdot(i, j, \phi, K)}$ and $w \leq_{i, v, H} w^{\prime}$.

The meaning of the definition is as follows: $w$ is at least as plausible as $w^{\prime}$ for $i$ at $(v, H)$ when the event $(m, j, \phi, K)$ occurs iff (I.) $(v, H \cdot(m, j, \phi, K))$ is already illegal (and therefore, all worlds are implausible from the point of view at $(v, H \cdot(m, j, \phi, K))$ ) or (II.) $i$ cannot distinguish $(v, H \cdot(m, j, \phi, K))$ with $(v, H)$ because of $m \neq i$ or (III.a.) $w^{\prime}$ is implausible at $(v, H \cdot(m, j, \phi, K))$ or (III.b.) $w$ is at least as plausible as $w^{\prime}$ for $i$ at the previous point $(v, H)$. Thus, in the case of (I.) and (III.a.), any worlds become implausible, and in the case of (II.), the preference relation is not changed, and therefore only the case of (III.b.) requires belief revision by the knowledge.

We defined a revision operation for the preference relation by another agent's knowledge. We regard worlds that contradict the knowledge as implausible, when we incorporate the information that $j$ believes $\phi$ into $i$ 's belief. However, nothing prevents worlds that satisfy $\neg \phi$ from becoming more plausible again upon learning new information as a result of future communications. Thus, we employ the moderate approach for the definition of revision by another agent's belief, because belief may be false and can not eliminate the information that is not known to be impossible. 
The basic idea can also be characterized by systems of spheres like the right side of figure 1. Change of preference relation by belief is explained as follows: the most plausible worlds that satisfy $\phi$ (worlds of part 1 in the figure) become the most plausible world in the next step of accepting the information that tells that $\phi$ is known, the secondmost plausible worlds that satisfy $\phi$ (worlds of part 2 in the figure) become the secondmost plausible worlds in the next step, the most plausible worlds that do not satisfy $\phi$ (worlds of part 3 in the figure) become the third-most plausible worlds in the next step, the second-most plausible worlds that do not satisfy $\phi$ (worlds of part 4) become the fourth-most plausible worlds, and the third-most plausible worlds that do not satisfy $\phi$ (worlds of part 5) become the fifth-most plausible worlds, but implausible worlds (marked black) remain implausible.

Again, $w \leq_{i, v, H \cdot(m, j, \phi, B)} w^{\prime}$ iff

I. $v, H \not \neq_{\mathcal{M}} L$ or $(m, j) \notin E$ or

II. $m \neq i$ and $w \leq_{i, v, H} w^{\prime}$ or

III. $m=i$ and

a. $w^{\prime} \in I m_{i, v, H}$ or

b. $w \notin I m_{i, v, H}$ and

1. $w, H \models_{\mathcal{M}} \phi, w^{\prime}, H \models_{\mathcal{M}} \phi$, and $w \leq_{i, v, H} w^{\prime}$, or

2. $w, H==_{\mathcal{M}} \phi, w^{\prime}, H \mid \neq_{\mathcal{M}} \phi$, or

3. $w, H \mid \neq_{\mathcal{M}} \phi, w^{\prime}, H \neq_{\mathcal{M}} \phi$, and $w \leq_{i, v, H} w^{\prime}$.

The meaning of the definition is as follows: $w$ is at least as plausible as $w^{\prime}$ for $i$ at $(v, H)$ when the event $(m, j, \phi, B)$ occurs iff (I.) $(v, H \cdot(m, j, \phi, B))$ is already not legal (and therefore, all worlds are implausible from the point of view at $(v, H \cdot(m, j, \phi, B))$ ) or (II.) $i$ cannot distinguish $(v, H \cdot(m, j, \phi, B))$ from $(v, H)$ because of $m \neq i$ or (III.a.) $w^{\prime}$ is implausible at $(v, H \cdot(m, j, \phi, B))$ (i.e., at $\left.(v, H)\right)$ or (III.b.1.) both $w$ and $w^{\prime}$ satisfy $\phi$ and $w$ is at least as plausible as $w^{\prime}$ for $i$ at the previous point $(v, H)$ or (III.b.2.) $w$ satisfies $\phi$ but $w^{\prime}$ does not satisfy $\phi$ or (III.b.3.) neither $w$ nor $w^{\prime}$ satisfy $\phi$ and $w$ is at least as plausible as $w^{\prime}$ for $i$ at the previous point $(v, H)$. Like the revision for knowledge, in the case of (I.) and (III.a.), any worlds become implausible, and in the case of (II.), the preference relation is not changed, and therefore, the case of (III.b.1.) - (III.b.3) is a standard condition for belief revision by knowledge.

While we assumed that $\leq_{i, w, \epsilon}=\leq_{i, v, \epsilon}$ when $\left.w\right|_{A t_{i}}=\left.v\right|_{A t_{i}}$, this assumption now can be generalized by the following theorem.

Theorem 1. If $w, H \models \models_{\mathcal{M}} L$ and $v, H^{\prime} \models_{\mathcal{M}} L$ and $(w, H) \sim_{i}\left(v, H^{\prime}\right)$, then $\leq_{i, w, H}=\leq_{i, v, H^{\prime}}$.

Proof. The proof is separated into two cases: (i) $w, H \cdot(m, j, \phi, e) \models_{\mathcal{M}} L$ and $m \neq i$ and $v, H^{\prime} \models_{\mathcal{M}} L$ and $(w, H \cdot(m, j, \phi, e)) \sim\left(v, H^{\prime}\right)$, and (ii) $w, H \cdot(i, j, \phi, e) \models_{\mathcal{M}} L$ and $v, H^{\prime} \cdot(i, j, \phi, e) \models_{\mathcal{M}} L$ and $(w, H \cdot(i, j, \phi, e)) \sim\left(v, H^{\prime} \cdot(i, j, \phi, e)\right)$. Then, $w, H \models \mathcal{M} L$ and $v, H^{\prime} \models_{\mathcal{M}} L$ and $(w, H) \sim\left(v, H^{\prime}\right)$. In the case of (i), we can derive $w, H \models \mathcal{M} L$ and $(w, H) \sim\left(v, H^{\prime}\right)$, and the conclusion that we want to show is proved from $\leq_{i, w, H}=\leq_{i, v, H^{\prime}}$ by induction. In the case of (ii), after deriving $w, H \models \mathcal{M}$ $L$ and $v, H^{\prime} \models_{\mathcal{M}} L$ and $(w, H) \sim\left(v, H^{\prime}\right)$, we will conclude the theorem from $\leq_{i, w, H}=\leq_{i, v, H^{\prime}}$ and the definition of the rewriting rules for the preference relation. 


\section{Postulates}

In this section, we will construct rational postulates for our revision operation that follow AGM's paradigm [1] and show the representation theorem in the restricted communication graph, which states that the above operation is essentially equivalent with the postulates. Although some of them are changed from AGM's original definition, we will discuss the reason why our result is restricted, and we conclude that the result is due to the fact that AGM's definition neglects the case that an external input comes from the other's knowledge.

First, we revisit AGM rational postulates for a belief revision operator $\dot{+}$ that accepts a belief set and a formula, and returns a belief set. Given some consequence relation $\vdash$, belief set $K$ is a set of sentences such that $K=\{\phi \mid K \vdash \phi\}$, i.e., logically closed set. Moreover, suppose that $K+\phi=\{\psi \mid K \cup\{\phi\} \vdash \psi\}$.

AGM1. For any sentence $\phi$ and any belief set $K, K \dot{+} \phi$ is a belief set.

AGM2. $\phi \in K \dot{+} \phi$.

AGM3. $K \dot{+} \phi \subseteq K+\phi$.

AGM4. If $\neg \phi \notin K$, then $K+\phi \subseteq K \dot{+} \phi$.

AGM5. $K \dot{+} \phi=K_{\perp}$ iff $\vdash \neg \phi$.

AGM6. If $\vdash \phi \Leftrightarrow \psi$, then $K \dot{+} \phi=K \dot{+} \psi$.

AGM7. $K \dot{+}(\phi \wedge \psi) \subseteq(K \dot{+} \phi)+\psi$.

AGM8. If $\neg \psi \notin K \dot{+} \phi$, then $(K \dot{+} \phi)+\psi \subseteq K \dot{+}(\phi \wedge \psi)$.

As the details of AGM postulates are well explained in [1], we will not recapitulate them here. Instead, we will indicate a problem that arises when applying the AGM postulates to our formalism. That is, the beliefs in $K$ are treated uniformly, with no distinction between truths and mere beliefs. Thus, an agent can not compare the knowledge that $\phi$ is implausible with the belief that $\phi$ is implausible. He cannot accept the information that $\phi$ is correct in the former case, while he can do so in the latter case. However, when we utilize the framework of belief set $K$, such a difference is neglected. In other words, an agent can not reject an input $\phi$, even though he knows $\neg \phi$. For this reason we introduce a variation of the AGM postulates that addresses this problem.

1. If $(w, H) \sim_{i}\left(v, H^{\prime}\right)$, then $w, H==_{\mathcal{M}} B_{i} \phi$ iff $v, H^{\prime} \models_{\mathcal{M}} B_{i} \phi$.

2. If $w, H \mid=_{\mathcal{M}} \neg C_{i} \neg \phi$, then $w, H \cdot(i, j, \phi, e) \mid=_{\mathcal{M}} B_{i} \phi$.

3. If $w, H \models{ }_{\mathcal{M}} C_{i} \neg \phi$, then $w, H \cdot(i, j, \phi, e) \models_{\mathcal{M}} B_{i} \psi$ iff $w, H \models{ }_{\mathcal{M}} B_{i} \psi$.

4. If $w, H \cdot(i, j, \phi, e) \models_{\mathcal{M}} B_{i} \psi$, then $v, H^{\prime} \models \mathcal{M} B_{i}(\phi \Rightarrow \psi)$.

5. If $w, H \models{ }_{\mathcal{M}} \neg B_{i} \neg \phi$ and $w, H \models=_{\mathcal{M}} B_{i}(\phi \Rightarrow \psi)$, then $v, H^{\prime} \cdot(i, j, \phi, e) \models{ }_{\mathcal{M}} B_{i} \psi$.

6. $w, H \models \mathcal{M} \neg B_{i} L$ iff $w, H \models \mathcal{M} \neg B_{i} \phi$ for any $\phi$.

7. If $\models_{\mathcal{M}} \phi \Leftrightarrow \psi$, then $w, H \cdot(i, j, \phi, e) \models_{\mathcal{M}} B_{i} \chi$ iff $w, H \cdot(i, j, \psi, e) \models_{\mathcal{M}} B_{i} \chi$.

8. If $w, H \cdot(i, j, \phi \wedge \psi, e) \models_{\mathcal{M}} B_{i} \chi$, then $w, H \cdot(i, j, \phi, e) \models_{\mathcal{M}} B_{i}(\psi \Rightarrow \chi)$.

9. If $w, H \models \models_{\mathcal{M}} \neg C_{i} \neg \phi, w, H \cdot(i, j, \phi, e) \models_{\mathcal{M}} \neg B_{i} \neg \psi$ and $w, H \cdot(i, j, \phi, e) \models \models_{\mathcal{M}}$ $B_{i}(\psi \Rightarrow \chi)$, then $w, H \cdot(i, j, \phi \wedge \psi, e) \models \mathcal{M} B_{i} \chi$.

10 .

10.a. If $w, H \models \mathcal{M} \neg C_{i} \neg \phi, w, H \cdot(i, j, \phi, e) \models_{\mathcal{M}} \neg C_{i} \neg \psi$ and $\models_{\mathcal{M}} \neg(\phi \wedge \psi)$, then $w, H \cdot(i, j, \phi, e) \cdot\left(i, k, \psi, e^{\prime}\right) \models_{\mathcal{M}} B_{i} \chi$ iff $w, H \cdot(i, k, \psi, B) \models_{\mathcal{M}} B_{i} \chi$. 
10.b. Else if $w, H \models \models_{\mathcal{M}} \neg C_{i} \neg \phi, w, H \cdot(i, j, \phi, e) \models_{\mathcal{M}} \neg C_{i} \neg \psi$ and $\mid \neq{ }_{\mathcal{M}} \neg(\phi \wedge \psi)$, then $w, H \cdot(i, j, \phi, e) \cdot\left(i, k, \psi, e^{\prime}\right) \models_{\mathcal{M}} B_{i} \chi$ iff $w, H \cdot(i, k, \phi \wedge \psi, B) \models_{\mathcal{M}} B_{i} \chi$.

10.c. Else if $w, H \models \mathcal{M} C_{i} \neg \phi$ and $w, H \cdot(i, j, \phi, e) \models \mathcal{M} \neg C_{i} \neg \psi$, then $w, H$. $(i, j, \phi, e) \cdot\left(i, k, \psi, e^{\prime}\right) \models_{\mathcal{M}} B_{i} \chi$ iff $w, H \cdot(i, k, \psi, B) \models_{\mathcal{M}} B_{i} \chi$.

10.d. Else if $w, H \models \mathcal{M} \neg C_{i} \neg \phi$ and $w, H \cdot(i, j, \phi, e) \models_{\mathcal{M}} C_{i} \neg \psi$, then $w, H$. $(i, j, \phi, e) \cdot\left(i, k, \psi, e^{\prime}\right) \models_{\mathcal{M}} B_{i} \chi$ iff $w, H \cdot(i, k, \phi, B) \models_{\mathcal{M}} B_{i} \chi$.

10.e. Else if $w, H \models{ }_{\mathcal{M}} C_{i} \neg \phi$ and $w, H \cdot(i, j, \phi, e) \models_{\mathcal{M}} C_{i} \neg \psi, w, H \cdot(i, j, \phi, e) \cdot$ $(i, k, \psi, e) \models_{\mathcal{M}} B_{i} \chi$ iff $w, H \models \models_{\mathcal{M}} B_{i} \chi$.

The following mapping acts as the translation between our postulates and the AGM ones.

$$
\begin{gathered}
\phi \in K \text { iff } w, H \models_{\mathcal{M}} B_{i} \phi . \\
\psi \in K \dot{+} \phi \text { iff } w, H \cdot(i, j, \phi, e) \models_{\mathcal{M}} B_{i} \psi .
\end{gathered}
$$

Some of them (4., 5., 7. and 8.) are equivalent with AGM postulates (AGM3., 4., 6. and 7. $)^{7}$. Postulates 2. and 9. are almost the same as AGM2. and AGM8., but they presuppose that $i$ is not convinced that input $\phi$ is false, i.e., he considers that $\phi$ is possible. Otherwise, he neglects input $\phi$ by 3 . Generally, belief revision that can reject external input is called nonprioritized belief change [9]. In our study, the reason for eliminating external input is the conviction that it is impossible. When histories cannot be distinguished by agent $i, i$ 's belief is also not distinguished in postulate 1 .

Since our approach is the combination of radical and moderate approach, we have introduced Postulate 10 for iterated belief revision. At first, suppose that information $\phi$ arrives at the agent $i$. When $\phi$ is inconsistent with $i$ 's knowledge, we should neglect the information $\phi$ from the result of the iterated belief revision (10.c and 10.e). Next, suppose that $\psi$ has arrived at the agent $i$ consecutively after $\phi$. When $\psi$ is inconsistent with $i$ 's current knowledge, we should neglect this information $\psi$ from the result of the iterated belief revision (10.d). Otherwise, $\psi$ is consistent with $i$ 's current knowledge. If the new information $\psi$ is inconsistent with the old information $\phi$, the new information eliminates the old information, and the result of iterated belief revision becomes equal to the belief revision by the new information (10.a). Otherwise, the iterated belief revision becomes equal to the belief revision by the conjunction of the new information and the old information (10.b).

Now we show that the following theorem holds when communication graph is restricted.

Theorem 2. Suppose that $E$ is fully connected. If a communication graph model $\mathcal{M}$ satisfies the rewriting rules for the preference relation, then it also satisfies all the postulates.

Proof. 1., 2., 3., 4., 6., 7., and 8. are rather easy to show. The problematic cases are 5., 9., and 10 . Here, we only show the case of 5.

Let a communication graph model $\mathcal{M}=\langle\mathcal{G}, \boldsymbol{A t}, \mathcal{P}, W\rangle$ satisfy the rewriting rules for the preference relation. Suppose that $v, H^{\prime} \models_{\mathcal{M}} \neg B_{i} \neg \phi$ and $v, H^{\prime} \models_{\mathcal{M}} B_{i}(\phi \Rightarrow$

\footnotetext{
${ }^{7}$ For 4., 5. and 8., note that $\psi \in K+\phi$ iff $\phi \Rightarrow \psi \in K$ by the deduction theorem.
} 


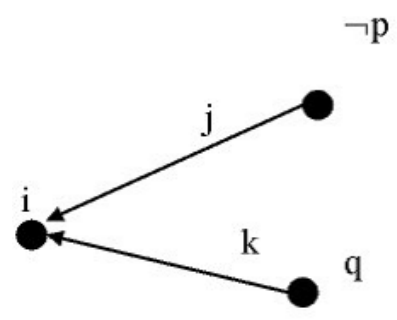

1

World $w$

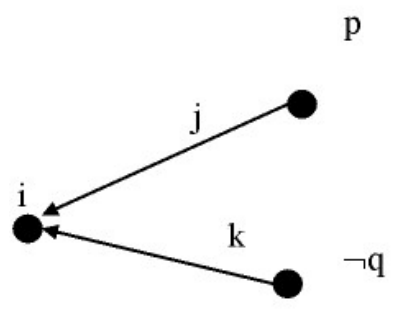

1

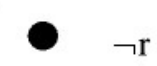

World $w^{\prime}$

Fig. 2. Example that does not satisfy postulate 5 .

$\psi)$. Then, there is some $(w, H)$ such that $(w, H) \sim_{i}\left(v, H^{\prime}\right)$ and $w, H=_{\mathcal{M}} L$, and for some $w^{\prime} \in \llbracket \phi \Rightarrow \psi \rrbracket_{i, w, H}^{\mathcal{M}}$, for all $w^{\prime \prime} \in \llbracket \neg(\phi \Rightarrow \psi) \rrbracket_{i, w, H}^{\mathcal{M}}, w^{\prime}<_{i, w, H} w^{\prime \prime}$. Besides, for all $w^{\prime} \in \llbracket \neg \phi \rrbracket_{i, w, H}^{\mathcal{M}}$, for some $w^{\prime \prime} \in \llbracket \phi \rrbracket_{i, w, H}^{\mathcal{M}}, w^{\prime \prime} \leq_{i, w, H} w^{\prime}$. Thus, for some $w^{\prime} \in \llbracket \phi \wedge \psi \rrbracket_{i, w, H}^{\mathcal{M}}$, for all $w^{\prime \prime} \in \llbracket \phi \wedge \neg \psi \rrbracket_{i, w, H}^{\mathcal{M}}, w^{\prime}<_{i, w, H} w^{\prime \prime}$. Therefore, for some $w^{\prime} \in \llbracket \psi \rrbracket_{i, w, H \cdot(i, j, \phi, e)}^{\mathcal{M}}$, for all $w^{\prime \prime} \in \llbracket \neg \psi \rrbracket_{i, w, H \cdot(i, j, \phi, e)}^{\mathcal{M}}, w^{\prime}<_{i, w, H \cdot(i, j, \phi, e)} w^{\prime \prime}$. (In the case of $e=K$, note that $\llbracket \phi \wedge \psi \rrbracket_{i, w, H}^{\mathcal{M}}=\llbracket K_{j} \phi \wedge \psi \rrbracket_{i, w, H}^{\mathcal{M}}$ and $\llbracket \phi \wedge \neg \psi \rrbracket_{i, w, H}^{\mathcal{M}}=$ $\left.\llbracket K_{j} \phi \wedge \neg \psi \rrbracket_{i, w, H}^{\mathcal{M}}.\right)$ Thus, we showed $v, H^{\prime} \cdot(i, j, \phi, e) \models_{\mathcal{M}} B i \psi$.

9 and 10 can be shown in a similar way.

Inconveniently, this theorem cannot be generalized to the unrestricted communication graph. Suppose that $\mathcal{M}=\langle\{\{i, j, k, l\},\{(i, j),(i, k)\}\},\langle\emptyset,\{p\},\{q\}\{r\}\rangle, \mathcal{P}, W\rangle$, where $\mathcal{P}$ is arbitrary except for some point $(v, \epsilon)$ for agent $i$. We define the preference relation in $(v, \epsilon)$ for agent $i$ as follows: let $w, w^{\prime} \in W$ such that $w(p)=0$, $w(q)=1$, and $w(r)=1$ (see the left side of figure 2), and $w^{\prime}(p)=1, w^{\prime}(q)=0$, and $w(r)=0$ (see the right side of figure 2), and suppose that $w$ is more plausible than $w^{\prime}$ (i.e., $w<_{i, v, \epsilon} w^{\prime}$ ) and for any $w^{\prime \prime} \in W$, if $w^{\prime \prime}$ is equal to neither $w$ or $w^{\prime}$, then $w^{\prime}$ is more plausible than $w^{\prime \prime}$ (i.e., $\left.w^{\prime}<_{i, v, \epsilon} w^{\prime \prime}\right)$. Then, $v, \epsilon \models_{\mathcal{M}} \neg B_{i} \neg(p \vee q)$ and $v, \epsilon=_{\mathcal{M}} B_{i}((p \vee q) \Rightarrow r)$, but $v,(i, j, p \vee q, K) \models_{\mathcal{M}} \neg B_{i} r$. The communication graph in this example is not fully connected, and therefore, postulate 5 is violated.

We can show the converse of the above theorem when the communication graph is restricted.

Theorem 3. Suppose that $E$ is existentially connected. If a communication graph model $\mathcal{M}$ satisfies all the postulates, then there is some model $\mathcal{M}^{\prime}$ that satisfies the rewriting rules for the preference relation, such that for any $w, H$ and $\phi, w, H \models_{\mathcal{M}} \phi$ iff $w, H \models \mathcal{M}^{\prime} \phi$.

Proof. Suppose form $(w)=\bigwedge\{p \in A t \mid w(p)=1\} \wedge \bigwedge\{\neg p \in A t \mid w(p)=0\}$. Given $\mathcal{M}=\langle\mathcal{G}, \boldsymbol{A t}, \mathcal{P}, W\rangle$, suppose $\mathcal{M}^{\prime}=\left\langle\mathcal{G}, \boldsymbol{A t}, \mathcal{P}^{\prime}, W\right\rangle$, where $\mathcal{P}^{\prime}{ }_{i}(v, H)=\leq_{i, v, H}$ such 
that

$$
\begin{gathered}
w \leq_{i, v, H} w^{\prime} \text { iff } v, H \mid \neq_{\mathcal{M}} L \text { or } w^{\prime} \in \operatorname{Im}_{i, v, H} \\
\text { or } v, H \models_{\mathcal{M}} C_{i} \neg\left(\operatorname{form}(w) \vee \operatorname{form}\left(w^{\prime}\right)\right) \\
\quad \text { or for some } j, \\
v, H \cdot\left(i, j, f \operatorname{form}(w) \vee \operatorname{form}\left(w^{\prime}\right), B\right) \models_{\mathcal{M}} \neg B_{i} \neg \operatorname{form}(w) .
\end{gathered}
$$

We will show that for any $w, H$, and $\phi, w, H \models_{\mathcal{M}} \phi$ iff $w, H \models_{\mathcal{M}^{\prime}} \phi$. It suffices to show that for any $w, H, \phi$, and $\psi, w, H=\models_{\mathcal{M}} \phi \rightarrow_{i} \psi$ iff $w, H \models_{\mathcal{M}^{\prime}} \phi \rightarrow_{i} \psi$, when $w, H \models_{\mathcal{M}} L$. It is obvious as follows.

$v, H \models \mathcal{M}^{\prime} \phi \rightarrow_{i} \psi$

$\mathbb{1}$

for some $w \in \llbracket \phi \wedge \psi \rrbracket_{i, v, H}^{\mathcal{M}^{\prime}}$, for all $w \in \llbracket \phi \wedge \neg \psi \rrbracket_{i, v, H}^{\mathcal{M}^{\prime}}, w<_{i, v, H} w^{\prime}$.

$\mathbb{1}$ (Note that $E$ is existentially connected.)

for some $w \in \llbracket \phi \wedge \psi \rrbracket_{i, v, H}^{\mathcal{M}}$, for all $w \in \llbracket \phi \wedge \neg \psi \rrbracket_{i, v, H}^{\mathcal{M}}$, for some $j$,

$v, H \cdot\left(i, j, f \operatorname{form}(w) \vee \operatorname{form}\left(w^{\prime}\right), B\right) \models_{\mathcal{M}} B_{i} f \operatorname{form}(w)$.

$\mathbb{1}$ (Note that $E$ is existentially connected.)

for some $w \in \llbracket \phi \wedge \psi \rrbracket_{i, v, H}^{\mathcal{M}}$, for all $w \in \llbracket \phi \wedge \neg \psi \rrbracket_{i, v, H}^{\mathcal{M}}, w<_{i, v, H} w^{\prime}$. $\Uparrow$

$v, H \models \mathcal{M} \phi \rightarrow_{i} \psi$

Moreover, connectedness, transitivity, satisfiability of the rewriting rules for the preference relations can be shown in the same way of the proof of the semantic version for belief revision [10].

That is, we showed the following representation theorem in the restricted communication graph.

Theorem 4. Suppose that $\mathcal{A}$ has at least two agents and $E$ is fully connected. There is some communication graph model $\mathcal{M}$ satisfies all the postulates iff there is some communication graph model $\mathcal{M}^{\prime}$, which satisfies the rewriting rules for the preference relation, such that for any $w, H$ and $\phi, w, H \models_{\mathcal{M}} \phi$ iff $w, H \models_{\mathcal{M}^{\prime}} \phi$.

\section{Conclusion}

The traditional AGM paradigm of belief revision does not distinguish belief from knowledge in the way epistemic logic does. Even though AGM postulates are directly translated into the possible world semantics, each agent still cannot identify the world in which he lives actually $[8,10]$, i.e., there is no difference between belief and knowledge. The modal logic approach to belief revision had the same problem, because the modal operator for knowledge was not introduced, which is distinguished from the modal operator for belief [3].

As already discussed, Friedman and Halpern [5,6] defined a belief operator with the epistemic logic of multiagent systems in Fagin et al. [4]. They considered that an agent $i$ believed $\phi$ iff $i$ knew that $\phi$ was plausible. Thus, their approach to iterated belief revision can distinguish belief from knowledge. 
Our study obviously depends on their result, because Pacuit and Parikh's history is equivalent with systems of runs in Fagin et al. [4] (See [14]). However, there is an important difference w.r.t. the acquisition of knowledge. They assumed that an agent acquires knowledge by observations from the external environment, and the information cannot be false. Our approach considers that an agent acquires knowledge from another agent by communication, and the information may be false. Therefore, our approach requires legality of history.

In this paper, we proposed a belief revision model for two types of uncertain communication. At first, when an agent as information source considers that he knows the information, and we can consider that there is reliable communication, we tend to accept this information. Secondly, when the agent that is the source of the information is himself less than certain, we are inclined to believe him, but at the same time we are less than fully convinced. Thus, postulates for the model was introduced, and the restricted representation theorem was shown.

However, there are four problems remaining with our study. Firstly, when we do not assume a fully connected communication graph, it may not satisfy the postulates. In the above example, we showed that an agent $i$ may not believe $\psi$, when he believes that $\phi \Rightarrow \psi$, and $j$ informs that he knows $\phi$, although $i$ believes that $\phi$ is not known to $j$, but can be true in fact. In the AGM postulates, such a case is forbidden. AGM postulates do not refer to the information source of external inputs. So when the first agent accepts a piece of information $\phi$ that comes from the second agent's knowledge, he will not distinguish it from other information $\phi$ received from a third agent's knowledge. However, these two situations can be regarded as different in our logic. Therefore, we do not agree with this idea, and consider that their postulates should be rethought for the case of multiagent communication, e.g., instead of our postulate 5., we use the following postulate.

- If $w, H \models \models_{\mathcal{M}} \neg B_{i} \neg K_{j} \phi$ and $w, H \models_{\mathcal{M}} B_{i}(\phi \Rightarrow \psi)$, then $v, H^{\prime} \cdot(i, j, \phi, K) \models_{\mathcal{M}}$ $B_{i} \psi$.

- If $w, H \models \models_{\mathcal{M}} \neg B_{i} \neg \phi$ and $w, H \models_{\mathcal{M}} B_{i}(\phi \Rightarrow \psi)$, then $v,\left.H^{\prime} \cdot(i, j, \phi, B)\right|_{\mathcal{M}} B_{i} \psi$.

Our model satisfies this postulate generally. Construction of rational postulates for belief revision in multiagent communication will be our future subject.

Secondly, we do not consider legality of belief. Therefore, a communication event $(i, j, \phi, B)$ is not related with whether $j$ believes $\phi$ actually or not. Since we consider that $i$ can believe it even if it is $j$ 's lie, while $i$ cannot know $\phi$ when $j$ informs that $j$ knows $\phi$ but it is false in fact, we did not define the legality of belief. In future work, we will study the problem of the representation of legality.

Thirdly, our definition of various concepts (e.g., the rewriting rules for the preference relation) is complicated. We suspect that this problem is due to the introduction of a legality predicate in the object language. Perhaps we may need to change the object language and lift the concept of history legality to the meta-language.

Finally, we have presupposed that the agent have common knowledge as to which atomic propositions are known to whom. Also, we assumed that the belief should be revised by the moderate approach while knowledge by the radical one. Because we did not wish to make the formal machinery in this paper even more complex than it already is, we accepted these assumptions for now. In future work though ,we may well rethink 
some of those first principles and move to a different point in the tradeoff between expressive realism and the complexity of the model.

\section{References}

1. C.E. Alchourrón, P. Gärdenfors, and D. Makinson. On the logic of theory change: partial meet contraction and revision functions. Journal of Symbolic Logic, 50:510-530, 1985.

2. R. Audi. Epistemology: Second edition. Routledge, 2003.

3. C. Boutilier. Unifying default reasoning and belief revision in a modal framework. Artificial Intelligence, 68(1):33-85, 1994.

4. R. Fagin, J.Y. Halpern, Y. Moses, and M.Y. Vardi. Reasoning about knowledge. The MIT Press, 1995.

5. N. Friedman and J.Y. Halpern. Modeling belief in dynamic systems, part i: Foundations. Artificial Intelligence, 95(2):257-316, 1997.

6. N. Friedman and J.Y. Halpern. Modeling belief in dynamic systems, part ii: Revision and update. Journal of Artificial Intelligence Research, 10:117-167, 1999.

7. P. Gärdenfors. Knowledge in flux: Modeling the dynamics of epistemic states. The MIT Press, 1988.

8. A. Grove. Two modelings for theory change. Journal of Philosophical Logic, 17:157-170, 1988.

9. S.O. Hansson. A textbook of belief dynamics. Kluwer Academic Publishers, 1999.

10. H. Katsuno and A. Mendelzon. Propositional knowledge base revision and minimal change. Artificial Intelligence, 52:263-294, 1991.

11. S. Kraus and D. Lehman. Knowledge, belief, and time. Theoretical Computer Science, 58:155-174, 1988.

12. S. Kraus, D. Lehman, and M. Magidor. Nonmonotonic reasoning, preferential models and cumulative logics. Artificial Intelligence, 44:167-207, 1990.

13. D. Lehman and M. Magidor. What does a conditional knowledge base entail? Artificial Intelligence, 55:1-60, 1992.

14. E. Pacuit. Some comments on history based structures. Journal of Applied Logic, 5:613-624, 2007.

15. E. Pacuit and R. Parikh. Reasoning about communication graphs. Augustus de Morgan Workshop: Interactive Logic: Games and Social Software, 2006.

16. H. Rott. Coherence and conservatism in the dynamics of belief ii: Iterated belief change without dispositional coherence. Journal of Logic and Computation, 13(1):111-145, 2003. 